\title{
Systematic Literature Review on Coronavirus Infectious Disease-19 and Dental Masks
}

\author{
$\mathrm{Ji}-$ Hyun $\mathrm{Kim}^{1}$ and Min-Young $\mathrm{Kim}^{2, \dagger}$ \\ 'Department of Dental Hygiene, Jeonbuk Science College, Jeongeup 56204, ${ }^{2}$ Department of Dental Hygiene, \\ Howon University, Gunsan 54058, Korea
}

\begin{abstract}
Background: Severe acute respiratory syndrome coronavirus 2 (SARS-CoV-2) is transmitted primarily through droplets, and dental practitioners are at risk of occupational exposure to SARS-CoV-2 infection owing to direct contact with the patient's mouth, aerosols from dental procedures, and saliva. Wearing a mask is believed to be the best method of protection against infection, and a systematic literature review was conducted on whether the dental masks used in dentistry are effective in preventing Coronavirus Infectious Disease-19 (COVID-19).

Methods: We used PubMed, Google Scholar, DBpia, NDSL, and KISS databases for this study. Of the 917 documents narrowed down by the search terms "Coronavirus, COVID-19, and dental," 83 documents were collected and studied. Ultimately, 42 of these papers were selected for analysis after considering duplication from the flow chart of the literature selection process. Results: While dental masks are often used when treating patients with unknown COVID-19 status, the Ministry of Food and Drug Safety stated that the use of dental masks is insufficient in preventing cross-transmission of COVID-19; instead, it was recommended that N95 masks, which correspond to KF94 masks, should be worn daily. On the other hand, wearing a dental mask and following precautions such as hand hygiene may not pose a significantly higher risk of infection than wearing an N95 mask when treating COVID-19 patients.

Conclusion: There is an ongoing discussion regarding the use of dental masks when treating dental patients, and many argue that different types of masks should be selected according to the degree of infection and the individual's respiratory condition. By considering the safety and efficacy of dental masks in preventing infection, improvements can be made in the management of COVID-19 and dental-related infections.
\end{abstract}

Key Words: COVID-19, Dental mask, SARS-CoV-2

\section{Introduction}

The coronavirus infection was first reported in Wuhan, Hubei Province, China in December 2019 ${ }^{1)}$. On February 11, 2020, the World Health Organization (WHO) announced the official name of this new infection as Coronavirus Infectious Disease-19 (COVID-19) ${ }^{2)}$. The pathogen behind COVID-19 is severe acute respiratory syndrome coronavirus 2 (SARS-CoV-2), which is similar to the virus that causes severe acute respiratory syndrome. Continuous monitoring of infected patients is necessary ${ }^{3,4)}$.
Transmission of COVID-19 occurs directly through droplets discharged during coughing, sneezing, and vocalization, and indirectly by touching mucous membranes, such as the oral cavity, nasal cavity, and eyes, after physical contact with contaminated surfaces ${ }^{5,6)}$. The incubation period may exceed 14 days, and patients may remain contagious up to three weeks after recovery ${ }^{4)}$.

Considering the possibility of COVID-19 transmission, medical professionals, particularly dental professionals working in dental clinics, are at a high risk of contracting the virus ${ }^{7)}$. There is a high biological risk of SARS-CoV-2 
transmission when carrying out dental procedures due to airborne droplets, aerosols generated by dental procedures, saliva, blood particles, and secretions, among others ${ }^{8)}$. Furthermore, COVID-19 transmission through droplets can occur when the distance between two people is less than $1 \mathrm{~m}$ apart. Dental care workers are vulnerable to cross-transmission of COVID-19 due to long treatment durations and the short (less than $1 \mathrm{~m}$ ) distance between the practitioner and the patient ${ }^{9)}$.

Given the mode of transmission, masks are an effective way to prevent infection ${ }^{4)}$. The types and classifications of masks vary by country. The N95, N99, N100, and R classifications are used in the United States, while European standards classify the filtering capability of masks into FFP1, FFP2, FFP3, P1, P2, and P3. The N95, N99, and N100 masks have filtration efficiencies of 95\%, $99 \%$, and $99.97 \%$ or more, respectively, while the FFP1, FFP2, and FFP3 masks have filtration efficiencies of $80 \%$, $94 \%$, and 99\%, respectively. Since N95 and FFP2 masks are nearly identical, they should be used to prevent the transmission of infectious diseases ${ }^{10,11)}$. The letters "KF" in KF masks mean "Korea Filter," and the KF99 and KF94 masks block more than $99 \%$ and $94 \%$ of $0.4 \mu \mathrm{m}$-sized particles, respectively, while the KF80 blocks more than $80 \%$ of $0.6 \mu \mathrm{m}$-sized particles. As recommended by the Centers for Disease Control and Prevention (CDC), the Emergency Use Authorization (EUA) approved the use of face masks that cover the mouth and nose for health care personnel (HCP) in the healthcare environment and in all populations to prevent the spread and transmission of severe acute respiratory syndrome coronaviruses during SARS-COV-2. However, because regular face masks only filter approximately $24 \%$ of particles smaller than $2.0 \mu \mathrm{m}$, they cannot protect against viruses ${ }^{12}$. A surgical mask, which is a mask used by medical personnel, is also called a dental mask due to its wide use in dentistry. These masks are fluid-resistant, loose, easily disposable, and function as a physical barrier and are primarily intended for surgical use. However, surgical masks do not completely block the inhalation of airborne pathogens such as viruses. The N95 mask is a medical mask that blocks pollutants from the air with a special filter and is worn by medical staff who treat COVID-19 patients. One $\mu \mathrm{m}$ means 1 millionth of a meter, and these masks can block $95 \%$ of small viruses 0.02 to $0.2 \mu \mathrm{m}$ in size, thereby providing a level of protection against viruses and bacteria that is 8 to 12 times higher than other masks ${ }^{11)}$.

The continued community spread of COVID-19 can lead to unintended aerosol exposure from infected patients in dental practices ${ }^{13)}$. Due to the recent epidemic COVID19, dental clinics are at a higher risk of infection than other medical settings. Various preventive measures, such as disinfection, hand washing, and the use of masks, are being implemented to suppress the rapid spread of the virus; however, unlike other preventive measures, different experts have different opinions regarding what type of masks should be worn. Therefore, we would like to study the relationship between dental masks, the personal protective equipment (PPE) worn by dental personnel, and COVID-19 prevention.

\section{Research Methods}

\section{Literature and database search}

A literature search was performed using PubMed and Google Scholar for foreign papers, and the Academic Database Service Database Periodical Information Academic (DBpia), Research Information Sharing Service (RISS), National Digital Science Leaders (NDSL), and Korean Studies Information Sharing System (KISS) for domestic papers. From January 2020 to December 2020, we searched for articles published in domestic and foreign journals using the search terms "Coronavirus, COVID-19, and dental."

\section{Literature selection criteria and exclusion criteria}

Among the 917 papers narrowed down using the search terms, 241 articles remained after the first screening stage, which was conducted by reviewing the titles and abstracts of the documents according to the inclusion and exclusion criteria. In the second screening stage, we examined the full-text in detail and assessed for the degree of conformity with our criteria, duplication along with the flow chart of the literature selection process, and valid associations between the use of dental masks and COVID-19 prevention. 
This left 83 articles (39 overseas and 3 domestic 42 total) for further analysis. The final papers were selected after two researchers independently reviewed both the accepted and rejected articles; experts were consulted in cases of disagreement. Non-original articles, gray literature, research reports, dissertations, studies published in conference presentations, studies in which the original text could not be verified, and studies published in languages other than Korean or English were excluded (Fig. 1).

\section{Results}

\section{Characteristics of studies included in the review}

Table 1 shows the general characteristics of the 42 studies included in the final analysis. The proportion of studies published by country is as follows: Italy $=14.2 \%$ (6/42); USA=9.5\% (4/42); Korea $=9.5 \%$ (4/42); Iran, Saudi Arabia, Poland, and Singapore $=7.1 \%$ each $(3 / 42)$; Nigeria, UK, Pakistan, Peru, and Egypt $=4.8 \%$ each $(2 / 42)$; and Canada, Brazil, Serbia, Romania, Germany, and Netherlands $=2.4 \%$ each $(1 / 42)$. The most common type of research included in the final analysis were theoretical review articles $(45.2 \%, 19 / 42)$ related to the research topic.

\section{Personal protective equipment masks suitable for COVID-19}

On February 5, 2020, the Central Disease Control Headquarters $(\mathrm{CDCH})$ recommended that healthcare

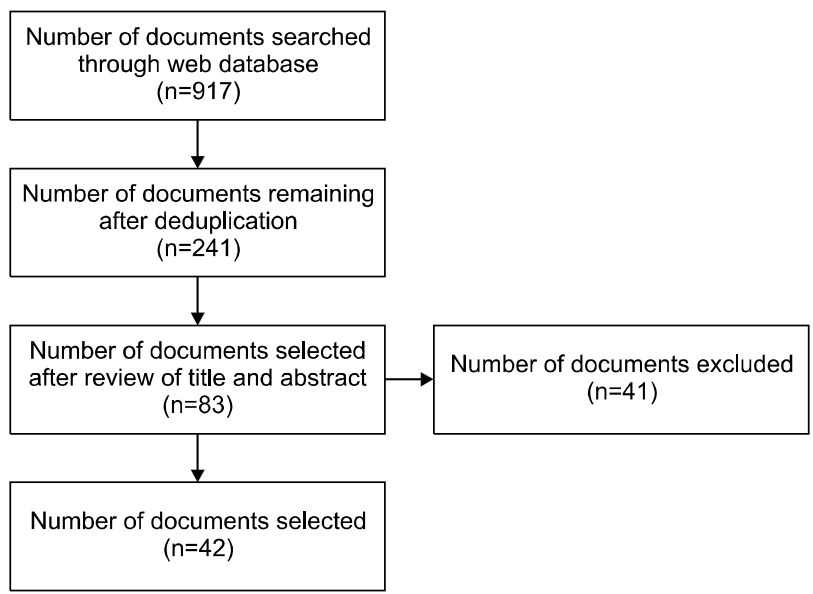

Fig. 1. Flow chart of the study selection process. personnel use KF94 and KF99 masks to protect the respiratory system from infection ${ }^{14)}$. Based on the European FFP-class system, the KF80, KF94, and KF99 masks perform almost the same functions as the FFP1, FFP2, and FFP3 masks, respectively. According to the National Institute for Occupational Safety and Health (NIOSH) criteria, N95 masks correspond to KF94 masks by the Ministry of Food and Drug Safety ${ }^{15}$.

The use of PPE is recommended when performing both aerosol-generating and -non- generating procedures in all dental practices. The use of PPE, such as N95, FFP2, or FFP3 masks, gloves, gowns, and protective glasses is the most important aspect of all dental procedures ${ }^{16)}$. In addition to the use of PPE, hand hygiene and disinfection of all surfaces in the clinic are also recommended as infection control measures in dentistry ${ }^{17)}$.

In their guidelines for COVID-19, the WHO recommended the use of NIOSH-certified N95, European Unioncertified FFP2, or other equivalent masks when performing procedures in dentistry ${ }^{18)}$. The types of masks recommended by the WHO and their filtration effects are

Table 1. Characteristics of Studies Included in the Review $(n=42)$

\begin{tabular}{clcc}
\hline & Characteristic & $\begin{array}{c}\text { Number of } \\
\text { studies }\end{array}$ & Percentage \\
\hline Nation of & Italy & 6 & 14.2 \\
publication & USA & 4 & 9.5 \\
& Korea & 4 & 9.5 \\
& Saudi Arabia & 3 & 7.1 \\
& Iran & 3 & 7.1 \\
& Poland & 3 & 7.1 \\
& Singapore & 3 & 7.1 \\
& Nigeria & 2 & 4.8 \\
& UK & 2 & 4.8 \\
& Pakistan & 2 & 4.8 \\
& Peru & 2 & 4.8 \\
& Egypt & 2 & 4.8 \\
& Canada & 1 & 2.4 \\
& Brazil & 1 & 2.4 \\
& Serbia & 1 & 2.4 \\
& Romania & 1 & 2.4 \\
& Germany & 1 & 2.4 \\
Study type & Netherlands & 1 & 2.4 \\
& Quantitative (survey) & 14 & 33.3 \\
& Policy research & 8 & 19.1 \\
& Review article & 19 & 45.2 \\
Others & 1 & 2.4 \\
\hline & & &
\end{tabular}


listed in Table $2^{19)}$.

The National Health Service (NHS) recommends the use of FFP3 masks in aerosol-generating processes ${ }^{20)}$. Regardless of the patient's condition, eyewear protection and an N95 mask should be used for all aerosol-generating procedures $^{4)}$. Some authors suggested wearing FFP2, FFP3, N95, or higher tier masks for aerosol-generating procedures, and the FFP1/dental mask for non-aerosolgenerating procedures ${ }^{21)}$.

In Germany, most dental personnel recommend the use of FFP2, FFP3, or N95 masks when treating patients regardless of the type of procedure and whether the presence or risk of infection is unknown or very high ${ }^{4)}$. When treating patients suspected to have COVID-19, dentists should wear full PPE consisting of an N95 mask, gown, gloves, and protective glasses ${ }^{22)}$. If treating a patient positive for COVID-19, an N95 (or higher) mask was recommended for all dental procedures ${ }^{16)}$. The FFP2, FFP3, and N95 masks provide dental practitioners with greater protection from viral respiratory infections than dental masks ${ }^{10)}$; in fact, FFP2 masks were found to be 100 times more effective than dental masks ${ }^{23}$. A survey of dental professionals in 26 countries on the use of masks found that most $(91.7 \%)$ used N95/FFP2 masks ${ }^{17)}$. However, only $12 \%$ were found to wear N95 masks ${ }^{24)}$. Although all dentists were mindful of the risk of COVID-19, they did not use N95 masks in the dental $\operatorname{clinic}^{25)}$.

With the advent of SARS-CoV-2, countries have 1) encouraged the general public to perform thorough hand hygiene; 2) banned public meetings in confined spaces; 3) advised avoidance of social contact; and 4) advocated the prevention of infection through the use of PPEs such as face masks and gloves ${ }^{26}$. In dental clinics, dental staff

Table 2. Type of Mask and Filtration Effect

\begin{tabular}{lcc}
\hline \multicolumn{1}{c}{ Mask type } & Filtration efficiency $(\%)$ & Particle size $(\mu \mathrm{m})$ \\
\hline FFP1 and P1 & $\geq 80$ & $\geq 0.3$ \\
FFP2 and P2 & $\geq 94$ & $\geq 0.3$ \\
N95 & $\geq 95$ & $\geq 0.3$ \\
N99 and FFP3 & $\geq 99$ & $\geq 0.023$ \\
P3 & $\geq 99.95$ & $\geq 0.3$ \\
N100 & $\geq 99.97$ & $\geq 0.02$ \\
\hline
\end{tabular}

should wear PPEs, such as a face mask, during patient examination and education ${ }^{27)}$. Long-term use or reuse of face masks is not recommended as contaminated masks increase the risk of infection ${ }^{28)}$. If contaminated with blood or other contaminants, the mask should be replaced and a new mask should be worn for up to 6 hours according to the CDC guidelines ${ }^{18)}$.

A study by Umeizudike et al. ${ }^{29)}$ emphasized that wearing face masks, gloves, and face shields during dental procedures could prevent COVID-19 transmission. Wearing a face mask alone reduces the risk of airborne infections in healthy people but provides insufficient protection from airborne viruses ${ }^{30)}$. Therefore, face mask recommendations are based on pragmatism, including availability and priority areas within the local healthcare system, rather than maximum protection ${ }^{31)}$.

The CDC and WHO recommended the use of dental masks when treating patients who have not been diagnosed with COVID-19 ${ }^{30)}$. Wearing a dental mask protects against fluids splashing into the respiratory tract from the nasal and oral cavities ${ }^{20)}$. The most frequently used PPEs in dental clinics in Italy were protective glasses $(90.55 \%)$, disposable gloves $(90.10 \%)$, and dental masks $(82.80 \%)^{26)}$. In dental clinics, dental personnel should wear dental masks, face shields, and protective eyewear during dental procedures $^{22)}$. However, it is not appropriate to use dental masks when performing dental procedures that generate aerosols ${ }^{18)}$. Dentists have reported that dental masks are insufficient in preventing cross-infection of COVID-19 and that N95 masks should be worn routinely ${ }^{30)}$. It has been reported that, while dental masks are recommended for office staff who do not directly handle patients, N95 masks should be used for staff who work in clinics with a unit chair ${ }^{20)}$.

\section{Discussion}

As COVID-19 began to spread rapidly to several countries, the WHO declared a global pandemic on March $11,2020^{32)}$. In a recently published paper, dental care during COVID-19 outbreaks was at high risk for transmission $^{8)}$. Among all medical professionals, the highest risk of infection was reported by dental practitioners ${ }^{4}$. 
Dental personnel risk occupational exposure to SARSCOV-2 infection due to contact with the patient's mouth, body fluids, and airborne pathogens ${ }^{30)}$. In addition, the significant amount of aerosol produced during dental procedures also contributes to the risk of COVID-19 infection ${ }^{9)}$. It is important for dental personnel to have appropriate PPE, including gowns, gloves, protective glasses, and masks; masks in particular provide a very important protective barrier ${ }^{33}$.

Most studies have shown that the N95 mask is more effective than the dental mask in protecting against COVID-19 infection ${ }^{34)}$. The most effective masks for protection against COVID-19, if infection in the patient is confirmed or suspected, are the N95, FFP2, and FFP3 masks, while the FFP2 and FFP3 masks are recommended for all aerosol-generating procedures performed in dentistry ${ }^{9}$.

Viral particles less than $2.0 \mu \mathrm{m}$ in size can penetrate various types of masks thereby leading to inhalation by the wearer. However, FFP2/N95 masks can reduce the risk of virus transmission by a factor of 1.5 to 2 times. N95 masks are often functionally differentiated respirators from masks. Regular use of FFP2 masks is not recommended or implemented because, while they reduce the average risk of pathogen transmission, they can also cause breathing difficulties when worn ${ }^{12}$. Although N95 respirators have shown several advantages over dental masks in several studies, in the clinical setting, insufficient data are available to demonstrate the superiority of N95 respirators over dental masks in protecting against infectious respiratory pathogens, including SARS-CoV- $2^{35)}$. It has been suggested that wearing a dental mask and following preventative measures such as hand hygiene may not pose a significantly higher risk of infection compared to wearing an N95 mask when treating COVID-19 patients ${ }^{36}$. Recent studies clearly demonstrate the effectiveness of dental masks in reducing respiratory virus excretion ${ }^{37}$.

There is a continuing debate about the effectiveness of dental masks against viruses and their transmission in dental patient care. Thus, biological safety rules in relevant occupations should be discussed, and possible guidance for more appropriate dental care should be provided. Preferentially, a mask should be chosen according to the degree of contamination and the individual's respiratory condition. While the use of dental masks is important, it is only one aspect of infection control; adherence to basic quarantine guidelines, such as rules on preventive behavior and social distancing, is also important. This study is meaningful since, by reviewing the certification criteria and the proper clinical applications of different types of masks, it demonstrates that the use of masks by dental personnel is a major means of preventing COVID-19 infection. In the future, additional research based on various domestic cases is needed to determine which masks are more effective in what situations.

\section{Notes}

\section{Conflict of interest}

No potential conflict of interest relevant to this article was reported.

\section{Ethical approval}

This study is a review-based study and does not require an IRB review.

\section{Author contributions}

Conceptualization: Min-Young Kim and Ji-Hyun Kim. Data acquisition: Min-Young Kim and Ji-Hyun Kim. Formal analysis: Min-Young Kim and Ji-Hyun Kim. Supervision: Min-Young Kim and Ji-Hyun Kim. Writingoriginal draft: Min-Young Kim and Ji-Hyun Kim. Writingreview \& editing: Min-Young Kim.

\section{ORCID}

Ji-Hyun Kim, https://orcid.org/0000-0002-7502-8118

Min-Young Kim, https://orcid.org/0000-0003-2095-8059

\section{Acknowledgements}

The authors would like to thank Dr. Jihyun Ahn for his helpful advice. This study was supported by a research fund from, Howon University.

\section{References}

1. Lu H, Stratton CW, Tang YW: Outbreak of pneumonia of 
unknown etiology in Wuhan, China: the mystery and the miracle. J Med Virol 92: 401-402, 2020.

https://doi.org/10.1002/jmv.25678

2. World Health Organization: Coronavirus disease (COVID-19) situation report 129. January 27, 2021, from: https://www. who.int/docs/default-source/coronaviruse/situation-reports/2 0200528-covid-19-sitrep-129.pdf?sfvrsn=5b154880_2(2020, May 28).

3. Rothan HA, Byrareddy SN: The epidemiology and pathogenesis of coronavirus disease (COVID-19) outbreak. J Autoimmun 109: 102433, 2020.

https://doi.org/10.1016/j.jaut.2020.102433

4. Gurzawska-Comis K, Becker K, Brunello G, Gurzawska A, Schwarz F: Recommendations for dental care during COVID-19 pandemic. J Clin Med 9: 1833, 2020.

https://doi.org/10.3390/jcm9061833

5. Secon H: Nearly 3,400 Chinese healthcare workers have gotten the coronavirus, and 13 have died. Retrieved January 25, 2021, from https://www.businessinsider.com/healthcareworkers-getting-coronavirus-500-infected-2020-2(2020, Mar 5).

6. Yu P, Zhu J, Zhang Z, Han Y: A familial cluster of infection associated with the 2019 novel coronavirus indicating possible person-to-person transmission during the incubation period. J Infect Dis 221: 1757-1761, 2020.

https://doi.org/10.1093/infdis/jiaa077

7. Isiekwe IG, Umeizudike KA, Daramola OO, Akeredolu MO, Leo-Olagbaye AA: The COVID-19 pandemic and dental residency training in Nigeria. Eur J Dent Educ 2021. [Epub ahead of print]

https://doi.org/10.1111/eje.12654

8. Peng X, Xu X, Li Y, Cheng L, Zhou X, Ren B: Transmission routes of 2019-nCoV and controls in dental practice. Int J Oral Sci 12: 9, 2020.

https://doi.org/10.1038/s41368-020-0075-9

9. Batista RCS, Arruda CVB, Cassimiro M, et al.: The role of the dental surgeon in controlling the dissemination of COVID-19: a literature review. ScientificWorldJournal 2020: 7945309, 2020.

https://doi.org/10.1155/2020/7945309

10. Villani FA, Aiuto R, Paglia L, Re D: COVID-19 and dentistry: prevention in dental practice, a literature review. Int J Environ Res Public Health 17: 4609, 2020. https://doi.org/10.3390/ijerph17124609
11. Melo Neto CLM, Bannwart LC, de Melo Moreno AL, Goiato MC: SARS-CoV-2 and dentistry-review. Eur J Dent 14(S1): S130-S139, 2020.

https://doi.org/10.1055/s-0040-1716438

12. Zemouri C, Awad SF, Volgenant CMC, Crielaard W, Laheij AMGA, de Soet JJ: Modeling of the transmission of coronaviruses, measles virus, influenza virus, Mycobacterium tuberculosis, and Legionella pneumophila in dental clinics. J Dent Res 99: 1192-1198, 2020. https://doi.org/10.1177/0022034520940288

13. Guo W, Chan BH, Chng CK, Shi AH: Two cases of inadvertent dental aerosol exposure to COVID-19 patients. Ann Acad Med Singap 49: 514-516, 2020. https://doi.org/10.47102/annals-acadmedsg.2020186

14. Ministry of Health and Welfare: Domestic occurrence of new coronavirus infections in Korean. Retrieved January 25, 2021, from http://www.mohw.go.kr/react/al/sal0301vw.jsp? PAR_MENU_ID $=04 \& M E N U \_I D=0403 \&$ page $=1 \& C O N T$ _ $\mathrm{SEQ}=352708$ (2020, February 5).

15. Ham SH, Choi WJ, Lee WH, Kang SK: Characteristics of health masks certified by the Ministry of Food and Drug Safety. J Environ Health Sci 45: 134-141, 2019. https://doi.org/10.5668/JEHS.2019.45.2.134

16. Brondani M, Cua D, Maragha T, et al.: A Pan-Canadian narrative review on the protocols for reopening dental services during the COVID-19 pandemic. BMC Oral Health 20: 352, 2020. https://doi.org/10.1186/s12903-020-01340-y

17. Ammar N, Aly NM, Folayan MO, et al.: Knowledge of dental academics about the COVID-19 pandemic: a multi-country online survey. BMC Med Educ 20: 399, 2020. https://doi.org/10.1186/s12909-020-02308-w

18. Centers for Disease Control and Prevention (CDC): Recommended guidance for extended use and limited reuse of N95 filtering facepiece respirators in healthcare settings. NIOSH. Retrieved January 28, 2021, from https://www.cdc. gov/niosh/topics/hcwcontrols/recommendedguidanceextuse. html(2020, March 27).

19. Bordea IR, Xhajanka E, Candrea S, et al.: Coronavirus (SARS-CoV-2) pandemic: future challenges for dental practitioners. Microorganisms 8: 1704, 2020. https://doi.org/10.3390/microorganisms8111704

20. Falahchai M, Babaee Hemmati Y, Hasanzade M: Dental care 
management during the COVID-19 outbreak. Spec Care Dentist 40: 539-548, 2020.

https://doi.org/10.1111/scd.12523

21. Becker K, Brunello G, Gurzawska-Comis K, et al.: Dental care during COVID-19 pandemic: survey of experts' opinion. Clin Oral Implants Res 31: 1253-1260, 2020.

https://doi.org/10.1111/clr.13676

22. Tay JRH, Ng E, Ong MMA, Sim C, Tan K, Seneviratne CJ: A risk-based approach to the COVID-19 pandemic: the experience in national dental centre Singapore. Front Med (Lausanne) 7: 562728, 2020.

https://doi.org/10.3389/fmed.2020.562728

23. Herron JBT, Hay-David AGC, Gilliam AD, Brennan PA: Personal protective equipment and COVID-19- a risk to healthcare staff? Br J Oral Maxillofac Surg 58: 500-502, 2020 .

https://doi.org/10.1016/j.bjoms.2020.04.015

24. Duruk G, Gümüşboğa ZŞ, Çolak C: Investigation of Turkish dentists' clinical attitudes and behaviors towards the COVID-19 pandemic: a survey study. Braz Oral Res 34: e054, 2020. https://doi.org/10.1590/1807-3107bor-2020.vol34.0054

25. Bontà G, Campus G, Cagetti MG: COVID-19 pandemic and dental hygienists in Italy: a questionnaire survey. BMC Health Serv Res 20: 994, 2020. https://doi.org/10.1186/s12913-020-05842-x

26. European Centre for Disease Prevention and Control (ECDC): Guidelines for the use of non-pharmaceutical measures to delay and mitigate the impact of 2019-nCoV. Retrieved January 25, 2021, from https://www.ecdc.europa. $\mathrm{eu} /$ sites/default/files/documents/novel-coronavirus-guideline s-non-pharmaceutical-measures_0(2020, February).

27. Mahdi SS, Ahmed Z, Allana R, et al.: Pivoting dental practice management during the COVID-19 pandemic-a systematic review. Medicina (Kaunas) 56: 644, 2020.

https://doi.org/10.3390/medicina56120644

28. Dacic SD, Miljkovic MN, Jovanovic MC: Dental care during the COVID-19 pandemic - to treat or not to treat? J Infect Dev Ctries 14: 1111-1116, 2020.

https://doi.org/10.3855/jidc. 13147

29. Umeizudike KA, Isiekwe IG, Fadeju AD, Akinboboye BO, Aladenika ET: Nigerian undergraduate dental students' knowledge, perception, and attitude to COVID-19 and infection control practices. J Dent Educ 85: 187-196, 2021.

https://doi.org/10.1002/jdd.12423

30. Tysiąc-Miśta M, Dubiel A, Brzoza K, Burek M, Pałkiewicz $\mathrm{K}$ : Air disinfection procedures in the dental office during the COVID-19 pandemic. Med Pr 72: 39-48, 2021. https://doi.org/10.13075/mp.5893.01005

31. Persoon IF, Stankiewicz N, Smith A, de Soet JJ, Volgenant CMC: A review of respiratory protection measures recommended in Europe for dental procedures during the COVID-19 pandemic. J Hosp Infect 106: 330-331, 2020. https://doi.org/10.1016/j.jhin.2020.07.027

32. World Health Organization: Coronavirus disease 2019 (COVID-19) situation report-51. Retrieved January 27, 2021, from https://www.who.int/docs/default-source/coronaviruse/ situation-reports/20200311-sitrep-51-covid-19.pdf?sfvrsn=1 ba62e57_10(2020, March 11).

33. Umer F, Haji Z, Zafar K: Role of respirators in controlling the spread of novel coronavirus (COVID-19) amongst dental healthcare providers: a review. Int Endod J 53: 1062-1067, 2020.

https://doi.org/10.1111/iej.13313

34. Ashtiani RE, Tehrani S, Revilla-León M, Zandinejad A: Reducing the risk of COVID-19 transmission in dental offices: a review. J Prosthodont 29: 739-745, 2020. https://doi.org/10.1111/jopr.13261

35. Bartoszko JJ, Farooqi MAM, Alhazzani W, Loeb M: Medical masks vs N95 respirators for preventing COVID-19 in healthcare workers: a systematic review and meta-analysis of randomized trials. Influenza Other Respir Viruses 14: 365-373, 2020. https://doi.org/10.1111/irv.12745

36. Ng K, Poon BH, Kiat Puar TH, et al.: COVID-19 and the risk to health care workers: a case report. Ann Intern Med 172: 766-767, 2020. https://doi.org/10.7326/L20-0175

37. Leung NHL, Chu DKW, Shiu EYC, et al.: Respiratory virus shedding in exhaled breath and efficacy of face masks. Nat Med 26: 676-680, 2020. https://doi.org/10.1038/s41591-020-0843-2 